\title{
APOIO SOCIAL E CONDIÇÓES DE SAÚDE DE IDOSOS BRASILEIROS DA COMUNIDADE
}

\author{
SOCIAL SUPPORT AND HEALTH CONDITIONS OF BRASILIAN \\ ELDERLY IN THE COMMUNITY
}

\section{APOYO SOCIAL Y CONDICIONES DE SALUD DE PERSONAS MAYORES BRASILEÑAS EN LA COMUNIDAD}

\author{
Darlene Mara Dos Santos Tavares* \\ Nayara Gomes Nunes Oliveira** \\ Pollyana Cristina Dos Santos Ferreira***
}

\begin{abstract}
RESUMO
Objetivos: descrever as características sociodemográficas e de saúde de idosos da comunidade; mensurar o apoio social dos idosos e verificar a associação entre as condições de saúde e o melhor nível de apoio social dos idosos. Material e Método: Estudo transversal desenvolvido na zona urbana da Microrregião de Saúde de Uberaba, Minas Gerais, com 962 idosos da comunidade; a coleta dos dados, por meio de entrevista direta, realizada no domicílio do idoso, de maio 2017 a junho 2018, foi desenvolvida por meio dos seguintes instrumentos: Escala de Depressão Geriátrica Abreviada, Versão Brasileira do Short Physical Performance Battery, Questionário Brasileiro de Avaliaçáo Funcional e Multidimensional, Componentes do fenótipo de fragilidade propostos por Fried, Questionário do Estudo Saúde Bem-estar e Envelhecimento e a Escala de Apoio Social. Resultados: A maioria era do sexo feminino, com 70-80 anos de idade, 1-5 anos de estudo, morava com companheiro, renda individual mensal de até um salário mínimo, desempenho físico moderado/bom, pré-frágil, sem problemas para dormir, com cinco ou mais morbidades autorreferidas e sem indicativo de sintomas depressivos; o percentual de idosos hospitalizados e que relataram a ocorrência de quedas, nos últimos 12 meses foi 17 e 28,3\%, respectivamente; a média do escore de apoio social foi de $88,14( \pm 16,81)$; associaram-se ao melhor nível de apoio social a não ocorrência de quedas nos últimos 12 meses $(\beta=0,064 ; p=0,045)$ e ausência do indicativo de sintomas depressivos $(\beta=0,254 ; p<0,001)$. Conclusóes: Os idosos com menores escores de sintomas depressivos e que náo tiveram quedas apresentaram melhor nível de apoio social.
\end{abstract}

Palavras-chave: Idoso; Apoio social; Saúde do idoso.

\footnotetext{
*Enfermeira. Doutora em Enfermagem. Departamento de Enfermagem em Educação e Saúde Comunitária, Universidade Federal do Triângulo Mineiro. Uberaba, Brasil. ORCID: http://orcid.org/0000-0001-9565-0476. E-mail: darlene.tavares@uftm.edu.br. Autora correspondente

**Enfermeira. Doutora em Atenção à Saúde. Pós-graduação em Atenção à Saúde, Universidade Federal do Triângulo Mineiro. Uberaba, Brasil. ORCID: http://orcid.org/0000-0003-4170-8761. E-mail: nayara.gomes06@yahoo.com.br.

***Enfermeira. Doutora em Atenção à Saúde. Departamento de Saúde Coletiva da Universidade Federal do Triângulo Mineiro. Uberaba, Brasil. ORCID: http://orcid.org/0000-0002-72383624. E-mail: pollyana.ferreira@uftm.edu.br.
} 


\begin{abstract}
Objectives: to describe the sociodemographic and health characteristics of the elderly population in the community; to measure the social support of the elderly and to verify the association between health conditions and the best level of social support. Material and Method: Cross-sectional study carried out in the urban area of health micro-region of Uberaba, State of Minas Gerais, with 962 elderly people from the community; data collection, through direct interview, carried out at the elderly's home, from May 2017 to June 2018, was developed using the following instruments: Abbreviated Geriatric Depression Scale, Brazilian Version of the Short Physical Performance Battery, Brazilian Functional and Multidimensional Assessment Questionnaire, Frailty Phenotype Components proposed by Fried, Health Well-Being Study Questionnaire and Aging and Social Support Scale. Results: Most were female, 70-80 years of age, had 1 to 5 years of schooling, lived with partner, single monthly income of up to one minimum wage, moderate / good physical performance, pre-frail, no problems for sleep, with five or more self-reported morbidities and no indication of depressive symptoms. The percentage of hospitalized elderly who reported falls in the last 12 months was $17 \%$ and $28.3 \%$, respectively. The mean social support score was $88.14( \pm 16.81)$. The absence of falls in the last 12 months $(\beta=0.064, p=0.045)$ and absence of the indicative of depressive symptoms $(\beta=0.254, p<0.001)$ were associated with the best level of social support. Conclusions: The elderly with lower scores of depressive symptoms and who did not have falls presented a better level of social support.
\end{abstract}

Key words: Aged; Social Support; Health of the Elderly.

\title{
RESUMEN
}

Objetivos: describir las características sociodemográficas y de salud de personas mayores de la comunidad; medir el apoyo social a las personas mayores y verificar la asociación entre las condiciones de salud y el mejor nivel de apoyo social. Material y Método: Estudio transversal desarrollado en la zona urbana de la microrregión de salud de Uberaba, Minas Gerais, con 962 personas mayores de la comunidad. La recolección de datos, a través de una entrevista directa, realizada en domicilio de los ancianos, desde mayo de 2017 hasta junio de 2018, se desarrolló utilizando los siguientes instrumentos: Escala de Depressão Geriátrica Abreviada, Versão Brasileira de la Short Physical Performance Battery, Cuestionario Brasilero de Evaluación Funcional y Multidimensional, Componentes de fenotipo de fragilidade propuestos por Fried, Cuestionario de Estudio Salud Bienestar y Envejecimiento y Escala de Apoyo social. Resultados: La mayoría eran mujeres, de 70 a 80 ańos de edad, 1 a 5 años de estudio, vivía con un compañero, una renta individual mensual de hasta un salario mínimo, un desempeño físico moderado / bueno, pre-frágil, sin problemas para dormir, con cinco o más morbilidades autorreferidas y sin indicativo de síntomas depresivos. El porcentaje de personas mayores hospitalizadas y que reportaron la ocurrencia de caídas, en los últimos 12 meses, fue el 17 y el 28,3\%, respectivamente. El promedio de la puntuación de apoyo social fue de $88,14( \pm 16,81)$. Se asociaron al mejor nivel de apoyo social a la no ocurrencia de caídas en los últimos 12 meses $(\beta=0,064 ; p=0,045)$ y ausencia del indicativo de síntomas depresivos $(\beta=0,254 ; p<0,001)$. Conclusiones: Las personas mayores con menores escores de síntomas depresivos y que no tuvieron caídas presentaron mejor nivel de apoyo social.

Palabras clave: Persona mayor; Apoyo Social; Salud del Anciano.

Fecha de recepción: 26/06/2019

Fecha de aceptación: 06/12/2019

\section{INTRODUÇÁO}

O processo de envelhecimento populacional tem ocorrido de maneira acelerada em diversos países, incluindo o Brasil ${ }^{(1)}$. No país, em 2019, o número de habitantes é de cerca de 210 milhóes. Desses, a proporção de pessoas com 60 anos ou mais corresponde a $13,35 \%$ da população. O Estado de Minas Gerais, regiâo onde está inserido o município em que foi realizado o presente estudo, segue essa 
tendência $(11,33 \%)$, contabilizando mais de três milhóes de pessoas nessa faixa etária ${ }^{(2)}$, justificando a necessidade de pesquisas que envolvam as especificidades dessa clientela.

Aliado a isso, estima-se que o número de idosos no país em 2040 seja mais que o dobro quando comparado ao ano de 2010, representando a proporção de 153 idosos para cada 100 jovens, cerca de $20 \%$ da população. Essa alteração no perfil demográfico acaba por refletir em novas demandas a serem enfrentadas pelo setor de saúde, visto que nessa faixa etária, de maneira geral, é possível identificar maior número de morbidades, de incapacidades para o desempenho de atividades diárias e a necessidade de acesso aos serviços mais especializados e de maior custo ${ }^{(1)}$.

Adicionalmente, observa-se um cenário desfavorável, no que se refere à estrutura familiar e à atuação dos diferentes segmentos sociais, frente às necessidades do idoso menos ativo e com algum tipo de dependência ${ }^{(1)}$. De tal contexto, emerge a reflexão da relação entre o apoio social ao idoso, considerando o processo de saúde e adoecimento.

O apoio social é compreendido como um construto multidimensional ${ }^{(3)}$, que envolve o processo em que se busca alcançar melhorias na qualidade de vida, a partir da otimização das oportunidades de saúde, participação e segurança ${ }^{(4)}$. Incluem variáveis como o vínculo social, a percepção do indivíduo sobre o universo social, o carinho, a assistência, o conforto e os recursos dos quais se pode dispor ${ }^{(3)}$.

Apesar de o apoio social ser considerado um fator preponderante para nova perspectiva de atenção à saúde do idoso, tem sido considerado um desafio na prática diária dos idosos, uma vez que deve contar com a sensibilização e participaçáo dos diversos atores sociais, como a família, os profissionais da saúde e do serviço social, amigos e o próprio idoso ${ }^{(5)}$.

Estudo de revisão bibliográfica verificou aumento do número de investigaçóes sobre o apoio social no Brasil, porém, relacionadas ao prognóstico, prolongamento do tempo de vida e bem estar de indivíduos acometidos por neoplasias e doenças crônicas, como diabetes mellitus, doenças cardiovasculares e transtornos mentais. Por outro lado, identificaram escassez em pesquisas que consideram os determinantes sociais de saúde e adoecimento e as condições de vida da população ${ }^{(3)}$, denotando a necessidade de pesquisas que ampliem o conhecimento nesta temática ${ }^{(6)}$. Nesse sentido, ressalta-se que para o profissional enfermeiro o conhecimento sobre as características dos idosos, envolvendo fatores sociais e considerando as especificidades locais, pode contribuir para o planejamento e implementação de ações de enfermagem, com destaque para aqueles que trabalham no contexto da saúde pública ${ }^{(7)}$.

Frente ao exposto, a pesquisa teve como objetivos descrever as características sociodemográficas e de saúde de idosos da comunidade; mensurar o apoio social dos idosos e verificar a associação entre as condiçôes de saúde e o melhor nível de apoio social.

\section{MATERIAL E MÉTODO}

Tipo de estudo: Trata-se de um estudo transversal com abordagem quantitativa, desenvolvido na zona urbana da Microrregião de Saúde de Uberaba, Minas Gerais.

Populaçáo e amostra: Destaca-se que essa microrregião é constituída por oito municípios que possuem $57 \%$ da população idosa da Macrorregião de Saúde do Triângulo Sul, sendo Uberaba referencia na atenção à saúde para a microrregião. Para a seleção da população da área urbana utilizouse a amostragem por conglomerado em múltiplo estágio. $\mathrm{O}$ cálculo do tamanho amostral considerou um coeficiente de determinação $\mathrm{R}^{2}=0,02 \mathrm{em}$ um modelo de regressão linear múltipla com sete preditores, tendo como nível de significância ou erro do tipo I de $\alpha=0,05$ e erro do tipo II de $\beta$ $=0,2$, resultando, portanto, em um poder estatístico apriorístico de 80\%. Utilizando-se o aplicativo PASS (Power Analysis and Sample Size), versão 13, introduzindo-se os valores acima descritos, obtevese uma amostra inicial de 977 idosos, mas deles, 15 apresentaram declínio cognitivo. Assim, a amostra do presente estudo foi composta por 962 idosos.

Critério de inclusão e exclusão: Foram incluídos no estudo os idosos com 60 anos ou mais de idade e que residiam na zona urbana da Microrregião de Saúde de Uberaba (MG); excluíram-se aqueles hospitalizados e/ou institucionalizados, com problemas de comunicação como surdez, não corrigida por aparelhos e transtornos graves da fala; com declínio cognitivo, avaliado pelo Miniexame 
do Estado Mental (MEEM). Considerou para a avaliação do MEEM os pontos de corte: $\leq 13$ para analfabetos, $\leq 18$ para escolaridade média (de um a 11 anos) e $\leq 26$ para alta escolaridade (superior a 11 anos) $)^{(8)}$.

Coleta dos dados: Foi realizada no domicílio dos idosos, de maio 2017 a junho 2018, por meio de entrevista direta. Para tal foram selecionados dez entrevistadores, os quais passaram por treinamento, capacitação e abordagem sobre questóes éticas da pesquisa.

Os dados sociodemográficos e econômicos foram obtidos por meio do formulário construído pelo Grupo de Pesquisa em Saúde Coletiva da Universidade Federal do Triângulo Mineiro (UFTM). Para avaliação das condições de saúde utilizaram-se os instrumentos descritos a seguir: Escala de Depressão Geriátrica Abreviada( ${ }^{(9)}$, para verificar a presença do indicativo de sintomas depressivos; Versão Brasileira do Short Physical Performance Battery ${ }^{(10)}$, para mensuração do desempenho físico; e os 26 itens contemplados no Questionário Brasileiro de Avaliação Funcional e Multidimensional $^{(11)}$, para identificação do número de morbidades autorreferidas. A condição de fragilidade foi identificada por meio dos cinco itens descritos como componentes do fenótipo de fragilidade, propostos por Fried et al. ${ }^{(12)}$, conforme segue: 1) perda de peso não intencional, avaliada por meio da seguinte pergunta: "No último ano, o(a) senhor (a) perdeu mais do que $4,5 \mathrm{~kg}$ ou $5 \%$ do peso corporal sem intenção?"; 2) autorrelato de exaustão e/ou fadiga, mensurado por duas questôes (itens 7 e 20) da versão brasileira da escala de depressão do Center for Epidemiologic Studies e os idosos que obtiverem escore 2 ou 3 em qualquer uma das questóes preencheram o critério de fragilidade para esse item ${ }^{(13)}$; 3) diminuição da força muscular, verificada com base na força de preensão palmar, por meio do dinamômetro hidráulico manual do tipo Jamar, modelo SAEHAN ${ }^{\circ}$ (SH5001-973), seguindo as recomendaçóes da American Society of Hand Therapists. Foram obtidas três medidas, apresentadas em quilograma/força (kgf), com um intervalo de um minuto entre elas, sendo considerado o valor médio dessas, adotando-se os pontos de corte propostos por Fried et al. ${ }^{(12)}$; 4) lentidão na velocidade de marcha, em que se considerou o tempo de marcha (em segundos) gasto para percorrer uma distância de 4,6 m. O idoso percorreu uma distância total de $8,6 \mathrm{~m}$, sendo os dois metros iniciais e os dois metros finais desconsiderados para o cálculo do tempo gasto na marcha. Foram realizadas três medidas, apresentadas em segundos, considerando-se o valor médio dessas. Utilizou-se como padrão o uso de um cronômetro profissional da marca Vollo ${ }^{\circ}$, modelo VL-1809 e os pontos de corte propostos por Fried et al. ${ }^{(12)}$; e 5) baixo nível de atividade física, verificado pela versão longa do Questionário Internacional de Atividade Física, adaptada para idosos ${ }^{(14)}$, sendo considerados ativos aqueles que despediam 150 min ou mais de atividade física semanal e inativos idosos que despediam de 0 a 149 min de atividade física semanal. Idosos com três ou mais desses itens foram classificados como frágeis, aqueles com um ou dois itens como pré-frágeis e aqueles com todos os testes negativos, não frágeis ${ }^{(12)}$.

A hospitalização e ocorrência de quedas nos últimos doze meses foram mensuradas por duas questóes pertencentes ao questionário do Estudo Saúde, Bem-estar e Envelhecimento, sendo elas: “ $O$ (a) Senhor (a) foi hospitalizado (a) nos últimos doze meses?" "O (a) Senhor (a) sofreu alguma queda nos últimos doze meses?” (15). A presença de problemas para dormir foi obtida por meio da pergunta elaborada pelas pesquisadoras: "O (a) Senhor (a) tem algum problema para dormir?”.

Para mensuraçáo do apoio social utilizou-se a Escala de Apoio Social ${ }^{(16)}$, sendo medido por meio da frequência que o idoso dispóe de apoio material, ou seja, a provisão de recursos práticos e materiais, como por exemplo, a ajuda no trabalho ou auxílio financeiro; interação social positiva/apoio afetivo que refletem na possibilidade de se ter alguém para realizar atividades de lazer e oferecer demonstraçôes físicas de amor e afeto; e o apoio emocional/ de informação, que consistem na habilidade da rede social em satisfazer as necessidades individuais em relação aos problemas emocionais, e o fato de poder contar com pessoas que aconselhem, informem e orientem $^{(16)}$. O escore final e, para cada uma das dimensóes, varia de 20 a 100 pontos, sendo que quanto maior o escore, melhor o nível de apoio social $^{(16)}$.

As variáveis sociodemográficas e econômicas estudadas foram: sexo (feminino; masculino), faixa etária, em anos $(60-70 ; 70-80 ; 80$ anos ou 
mais), escolaridade, em anos de estudo (0; 1-5; 5 ou mais), estado conjugal (com companheiro/a; sem companheiro/a) e renda individual mensal, em salários mínimo (sem rendimento; $\leq 1 ;>1$ ). Variáveis relacionadas à saúde: hospitalização nos últimos 12 meses (sim; não), ocorrência de quedas nos últimos 12 meses (sim; não), desempenho físico (incapacidade/baixo; moderado/bom), condição de fragilidade (não frágil; pré-frágil; frágil), problemas para dormir (sim; não); número de morbidades autorreferidas $(0-5 ; 5$ ou mais), indicativo de sintomas depressivos (sim; não), e apoio social (apoio material; interação social positiva/apoio afetivo; apoio emocional/ de informação).

Processamento e análise de dados: Construiuse um banco de dados eletrônico, no programa Excel $^{\circ}$, com dupla digitação. Após a verificação das inconsistências entre as duas bases de dados, o banco de dados foi importado para o software "Statistical Package for the Social Sciences" (SPSS) versão 22.0, para análise.

Os dados foram submetidos à análise de frequência absoluta e relativa para variáveis categóricas; e média e desvio padrão para as quantitativas. Para verificar as condiçôes de saúde associadas ao melhor nível de apoio social foi realizada análise bivariada preliminar empregandose o teste $t$ de Student $(p \leq 0,10)$. As variáveis de interesse que atenderam o critério estabelecido $(p \leq 0,10)$ foram dicotomizadas e introduzidas no modelo de regressão linear múltipla $(p<0,05)$. Considerou como desfecho o escore de apoio social e as variáveis de análise das condiçóes de saúde: hospitalização e ocorrência de quedas nos últimos 12 meses; desempenho físico; condição de fragilidade; problemas para dormir; número de morbidades autorreferidas e indicativo de sintomas depressivos.

O projeto foi aprovado pelo Comitê de Ética em Pesquisa com Seres Humanos da UFTM, protocolo $n^{\circ} 2.053 .520$. Após a anuência do idoso e a assinatura do Termo de Consentimento Livre Esclarecido, conduziu-se a entrevista.

\section{RESULTADOS}

A Tabela 1 apresenta as características sociodemográficas, econômica e as condiçôes de saúde dos idosos da Microrregiáo de Saúde de Uberaba (MG). Do total de idosos entrevistados (962) verificou-se que a maioria era do sexo feminino $(66,9 \%)$, com $70-80$ anos de idade $(41,4 \%)$, com 1-5 anos de estudo (52,1\%), morava com companheiro $(42,6 \%)$ e com renda individual mensal de até um salário mínimo (51,9\%). Entre os idosos, $17 \%$ foram hospitalizados e $28,3 \%$ relataram ocorrência de quedas nos últimos 12 meses. Predominaram idosos com desempenho físico moderado/bom $(75,8 \%)$, pré-frágil $(46,5 \%)$, sem problemas para dormir $(56,2 \%)$, com cinco ou mais morbidades autorreferidas $(68,2 \%)$ e sem indicativo de sintomas depressivos (76,3\%).

A Tabela 2 apresenta a distribuição das variáveis de condição de saúde, segundo escore de apoio social dos idosos da Microrregiáo de Saúde de Uberaba (MG). Em relaçáo à mensuração do apoio social, a média alcançada pelos idosos considerando-se as três dimensões foi de 88,14 $( \pm 16,81) . \mathrm{Na}$ análise de cada dimensão verificouse maior escore de apoio material $(92,03 \pm 17,36)$. As dimensóes apoio emocional/de informação $(86,91 \pm 19,71)$ e interação social positiva/apoio afetivo $(87,37 \pm 18,76)$ apresentaram escores abaixo da média total.

As variáveis da análise bivariada preliminar, inseridas no modelo multivariado, que atenderam ao critério adotado $(p \leq 0,10)$ foram: ocorrência de quedas nos últimos 12 meses $(p<0,001)$; desempenho físico $(p<0,001)$; condição de fragilidade $(p<0,001)$; problemas para dormir $(p=0,001)$ e indicativo de sintomas depressivos $(p<0,001)$.

A Tabela 3 apresenta a associação entre o apoio social e as condiçóes de saúde dos idosos da Microrregiáo de Saúde de Uberaba (MG). Associaram-se ao melhor nível de apoio social a náo ocorrência de quedas nos últimos 12 meses $(\beta=0,064 ; p=0,045)$ e a ausência do indicativo de sintomas depressivos $(\beta=0,254 ; p<0,001)$ (Tabela 3). 
Tabela 1. Distribuição de frequência das características sociodemográficas, econômica e das condiçóes de saúde dos idosos da Microrregião de Saúde de Uberaba, Minas Gerais, Brasil, 2018 (n=962).

\begin{tabular}{|c|c|c|c|}
\hline Variáveis & & $\mathbf{n}$ & $\%$ \\
\hline \multirow[t]{2}{*}{ Sexo } & Feminino & 644 & 66,9 \\
\hline & Masculino & 318 & 33,1 \\
\hline \multirow[t]{3}{*}{ Faixa etária } & $60-70$ & 359 & 37,3 \\
\hline & $70-80$ & 398 & 41,4 \\
\hline & 80 ou mais & 205 & 21,3 \\
\hline \multirow[t]{3}{*}{ Escolaridade } & Nenhum & 171 & 17,8 \\
\hline & $1-5$ & 501 & 52,1 \\
\hline & 5 ou mais & 290 & 30,1 \\
\hline \multirow[t]{4}{*}{ Estado conjugal } & Nunca morou com companheiro & 66 & 6,9 \\
\hline & Mora com o companheiro (a) & 410 & 42,6 \\
\hline & Viúvo & 378 & 39,3 \\
\hline & Separado (a)/desquitado (a)/divorciado (a) & 108 & 11,2 \\
\hline \multirow[t]{3}{*}{ Renda mensal individual } & Sem renda & 53 & 5,5 \\
\hline & Menor ou igual a um salário mínimo & 499 & 51,9 \\
\hline & Maior que um salário mínimo & 410 & 42,6 \\
\hline \multirow[t]{2}{*}{ Hospitalização últimos 12 meses } & Sim & 164 & 17 \\
\hline & Não & 798 & 83 \\
\hline \multirow[t]{2}{*}{ Ocorrência quedas últimos 12 meses } & Sim & 272 & 28,3 \\
\hline & Não & 690 & 71,7 \\
\hline \multirow[t]{2}{*}{ Desempenho físico } & Incapacidade/Baixo & 233 & 24,2 \\
\hline & Moderado/Bom & 729 & 75,8 \\
\hline \multirow[t]{3}{*}{ Condição de Fragilidade } & Não frágil & 274 & 28,5 \\
\hline & Pré-frágil & 451 & 46,5 \\
\hline & Frágil & 237 & 24,6 \\
\hline \multirow[t]{2}{*}{ Problema para dormir } & Sim & 421 & 43,8 \\
\hline & Não & 541 & 56,2 \\
\hline \multirow[t]{2}{*}{ Número morbidades autorreferidas } & $0-5$ & 306 & 31,8 \\
\hline & 5 ou mais & 656 & 68,2 \\
\hline \multirow[t]{2}{*}{ Indicativo sintomas depressivos } & Sim & 228 & 23,7 \\
\hline & Não & 734 & 76,3 \\
\hline
\end{tabular}


Tabela 2. Análise comparativa do escore de apoio social com as variáveis de condiçóes de saúde dos idosos da Microrregião de Saúde de Uberaba, Minas Gerais, Brasil, 2018 ( $\mathrm{n}=962)$.

\begin{tabular}{llcrc}
\hline Variáveis & & Média & Desvio padráo & $\boldsymbol{p}^{*}$ \\
\hline Hospitalização últimos 12 meses & Não & 88,38 & 16,55 & 0,334 \\
& Sim & 86,99 & 18,04 & \\
\hline Ocorrência quedas últimos 12 meses & Não & 89,63 & 15,56 & $<0,001$ \\
& Sim & 84,37 & 19,14 & \\
\hline Desempenho físico & Moderado/Bom & 89,41 & 15,48 & $<0,001$ \\
& Incapacidade/Baixo & 84,17 & 19,94 & \\
\hline Condição de Fragilidade & Náo fragilidade & 92,16 & 14,06 & $<0,001$ \\
& Pré-fragilidade/fragilidade & 86,55 & 17,54 & \\
\hline Problema para dormir & Náo & 89,79 & 15,25 & 0,001 \\
& Sim & 86,04 & 18,42 & \\
\hline Número de morbidades autorreferidas & $0-5$ & 89,28 & 16,36 & 0,153 \\
& 5 ou mais & 87,62 & 17 & \\
\hline Indicativo de sintomas depressivos & Náo & 90,98 & 13,76 & $<0,001$ \\
& Sim & 79 & 21,79 & \\
\hline
\end{tabular}

Nota: ${ }^{*} \mathrm{p} \leq 0,10$. Teste t de Student.

Tabela 3. Modelo final de regressão linear múltipla para o apoio social e as condiçôes de saúde dos idosos da Microrregião de Saúde de Uberaba, Minas Gerais, Brasil, 2018 (n=962).

\begin{tabular}{llll}
\hline Variáveis & & $\beta$ & $\boldsymbol{p}^{*}$ \\
\hline Ocorrência quedas últimos 12 meses & Sim & 1 & \\
& Não & 0,064 & 0,045 \\
\hline Desempenho físico & Incapacidade/ Baixo desempenho & 1 & \\
& Moderado/ Bom & 0,036 & 0,271 \\
\hline Condição de fragilidade & Pré-frágil/frágil & 1 & \\
& Não fragilidade & 0,064 & 0,053 \\
\hline Problemas para dormir & Sim & 1 & \\
& Não & 0,049 & 0,117 \\
\hline Indicativo de sintomas depressivos & Sim & 1 & \\
& Não & 0,254 & $<0,001$ \\
\hline
\end{tabular}

Nota: ${ }^{*} \mathrm{p}<0,05$; 1-Categoria de referência. 


\section{DISCUSSÁO}

Os resultados obtidos, em relação às características sociodemográficas e econômicas, estão em consonância com estudos nacionais e internacionais, os quais verificaram o predomínio de mulheres idosas ${ }^{(6,17)}$, faixa etária mais elevada ${ }^{(17)}$, vivendo com companheiro ${ }^{(18)}$, com baixos níveis de escolaridade $^{(6,17)}$ e de renda ${ }^{(17)}$.

$\mathrm{O}$ percentual de idosos que referiram terem sido hospitalizados nos últimos doze meses foi inferior quando comparado aos dados do Brasil, considerando o período de janeiro a agosto de 2018, no qual $25,58 \%$ do total de internaçóes corresponderam à faixa etária de 60 anos ou mais ${ }^{(19)}$. Os cuidados em saúde prestados aos idosos nesse nível de atenção são, normalmente, onerosos, além de terem maior potencial para a ocorrência de iatrogenias ${ }^{(20)}$, denotando a necessidade de uma rede de apoio que promova o acompanhamento da saúde de maneira eficaz, considerando suas especificidades e necessidades.

Em relação às quedas, pesquisa de base populacional realizada no Brasil identificou prevalência inferior ao obtido no presente estudo $(7,8 \%)$; contudo, investigaram as quedas no último ano que levaram o idoso a procurar o serviço de saúde ${ }^{(21)}$. Nesse sentido, pressupóe-se, que muitos dos idosos avaliados podem ter sofrido quedas e não terem buscado os serviços de saúde para avaliação.

Entre as repercussóes oriundas das quedas ressalta-se o maior acesso aos serviços de saúde ${ }^{(21)}$. Mediante esse quadro, é possível denotar a relevância do estabelecimento de redes de apoio micro e macrossocial que auxiliem o idoso caso necessitem, devido às circunstâncias relacionadas ao adoecimento.

Percentual inferior de desempenho físico moderado/bom foi evidenciado em pesquisa realizada com idosos de uma região do Rio Grande do Sul $(49,3 \%)^{(22)}$. Entretanto, referem-se à avaliação de indivíduos institucionalizados, o que possivelmente pode ter influenciado no resultado.

Consoante aos dados obtidos nessa pesquisa, estudo realizado com idosos no município de Sáo Paulo identificou percentual semelhante de indivíduos que apresentavam a condição de préfragilidade $(41,5 \%)^{(23)}$. Diante desses resultados, emerge a reflexão sobre as redes de apoio formais e informais destinada à populaçáo idosa, frente à necessidade de cuidados e de preservarem sua autonomia e bem-estar ${ }^{(24)}$.

$O$ percentual daqueles que mencionaram ter problemas para dormir foi congruente ao verificado em inquérito conduzido com idosos residentes em São Paulo $(44,9 \%)^{(25)}$. Adicionalmente, pesquisa realizada com idosos no Brasil identificou que a grande maioria dos participantes referia possuir alguma morbidade $(93,7 \%)^{(24)}$. Referente ao indicativo de depressão, resultados semelhantes foram obtidos em estudo realizado em São Paulo com adultos e idosos $(25,1 \%)^{(26)}$.

Nesse contexto, inquérito realizado na atenção básica demonstrou que pessoas que apresentam alguma doença física, principalmente em fases mais graves, tendem a ampliar a rede de apoio social e que aqueles que possuem suporte e estáo mais integrados à rede tendem a desenvolverem menos transtornos mentais comuns, como depressão(26). Assim, é possível refletir sobre a importância do apoio social para a manutenção ou restauração da saúde dos idosos, seja a partir do núcleo familiar ou de pessoas próximas, mas também considerando o acesso aos serviços de saúde e demais setores.

Em relação à mensuração do apoio social, escores inferiores foram verificados em estudos nacional $(74,43)^{(27)}$ e internacional $(70,22)^{(28)}$ desenvolvidos entre idosos da comunidade. Os profissionais de saúde, em especial os enfermeiros, durante o cuidado prestado aos idosos, devem considerar de forma integral as questôes que interferem na saúde, dentre elas o apoio social, que é tido como um fator de proteçâo para as boas condiçóes de saúde dessa população ${ }^{(5,29)}$. Sendo assim, abordagens que fortaleçam o apoio social e possibilitam integrar a família e componentes da rede social à assistência, são recursos que podem ser apreciados e utilizados, por agregar qualidade à saúde desses indivíduos ${ }^{(26)}$.

$\mathrm{Na}$ análise de cada dimensão, o melhor nível de apoio material também foi verificado em estudo nacional $^{(27)}$, ou seja, a disponibilidade de serviços práticos e recursos materiais, incluindo, por exemplo, ajuda no trabalho ou auxílio financeiro, é satisfatória para esses idosos. Contudo, percebe-se menos a possibilidade de terem com quem contar para dividir suas preocupaçóes e sentimentos; solicitar informaçóes e a existência de pessoas em sua rede social que lhes amem e com quem podem ter momentos de prazer e relaxamento ${ }^{(16)}$. Pesquisa internacional identificou maiores escores de apoio 
material entre idosos espanhóis, e apoio afetivo entre os holandeses. Contudo, destacou-se que os espanhóis apresentaram escores mais elevados de solidão ${ }^{(30)}$.

Por contribuir com melhorias nas condiçôes de saúde da população idosa ${ }^{(5)}$, o apoio social se enquadra como parte importante da atenção integral à saúde do idoso(5). Neste contexto, identificar o papel do apoio social no cuidado ao idoso possibilita verificar a forma como essa populaçáo interage com sua rede social, permitindo que outras pessoas tenham novas perspectivas sobre o cuidado, propiciando assim, tanto avanço do conhecimento científico, quanto para a sociedade como um todo ${ }^{(31)}$.

A avaliação do apoio social, além de indicar o quão integrado socialmente o idoso está, também identifica o grau com que as relaçôes interpessoais correspondem a determinadas funçóes e lhe oferecem suporte em momentos de crise ou readaptação ${ }^{(32)}$. O apoio social está relacionado a vários desfechos de saúde, sendo entendido como um fator que pode auxiliar no processo adaptativo às situaçóes de vida adversas ${ }^{(33)}$.

No presente estudo, os idosos que náo tiveram quedas nos últimos 12 meses apresentaram melhor nível de apoio social, resultado que náo condiz com pesquisa desenvolvida entre idosos da comunidade de Nova Iorque ${ }^{(30)}$. Destaca-se que não foram encontradas, na literatura nacional, investigaçóes que associaram o apoio social à ocorrência de quedas entre idosos da comunidade. $\mathrm{O}$ apoio social deve ser avaliado, uma vez que as informaçóes coletadas ajudarão os profissionais de saúde a traçar estratégias e intervençóes de promoção de saúde, prevenção e manejo de quedas, que auxiliaráo os idosos a se manterem independentes em suas $\operatorname{atividades}^{(34)}$.

O melhor nível de apoio social presente entre idosos sem indicativo de sintomas depressivos, verificado nessa pesquisa, diverge de estudo nacional $(p=0,068)^{(35)}$ e corrobora com investigação internacional ${ }^{(36)}$. Para o idoso, o apoio social é um fator essencial para se perceber saudável e a família assume um papel central na sua rede apoio, pois além de auxiliar nas práticas de autocuidado e prevençáo de agravos, que contribuem para uma melhor condiçáo de saúde, podem garantir a segurança contra os sentimentos de abandono ${ }^{(37)}$. $\mathrm{O}$ apoio social representado por amigos, vizinhos e grupos religiosos, também exerce papel fundamental, principalmente em momentos de dificuldade $^{(37)}$. Assim, intervençóes para combater e reduzir a presença de sintomas depressivos entre os idosos devem considerar o apoio social e seus efeitos sobre os comportamentos de saúde.

Estes dados sugerem a necessidade dos profissionais de saúde, em especial os enfermeiros, conhecerem sobre as necessidades dos idosos para implementar medidas sociais de promoção da saúde e prevenção de agravos. Além disso, os formuladores de políticas de públicas devem estabelecer programas que estimulem e favoreçam o apoio social dos idosos da comunidade ${ }^{(29)}$.

Ainda que não seja possível identificar a relação de causa e efeito entre as variáveis, visto que se trata de um estudo transversal, os resultados encontrados reforçam a necessidade de estímulo e manutenção do apoio social nesta população. A exclusão de idosos com comprometimento cognitivo pode favorecer uma amostra mais saudável. No entanto, a possibilidade de viés de seleção foi minimizada, uma vez que todos os idosos elegíveis foram entrevistados. Assim, futuras pesquisas multicêntricas e de coorte devem ser conduzidas, o que contribuiria para o planejamento de políticas de saúde para os idosos. Entretanto, a identificação das condiçóes de saúde associadas ao melhor nível de apoio social entre idosos da comunidade pode subsidiar o planejamento de açóes direcionadas ao contexto, de modo a nortear a atuação profissional.

\section{CONCLUSÃO}

Os resultados obtidos demonstram que em relação às características sociodemográficas os idosos acompanharam o perfil nacional, com predomínio de mulheres, com baixo nível de escolaridade e de renda, e que residem com companheiro.

Sobre as questóes de saúde, destaca-se o predomínio de idosos que relataram apresentar múltiplas morbidades, terem sofrido quedas no último ano, além daqueles que se encontram na condição de pré-fragilidade, podendo denotar lacunas na rede de atenção destinada ao atendimento dessa população, de forma a identificar precocemente os casos e, assim, estabelecer medidas de intervenção apropriadas.

Os menores escores identificados nas dimensôes 
de apoio emocional/de informação e interação social positiva/apoio afetivo apontam para a necessidade de um olhar ampliado às necessidades dos idosos avaliados, buscando reconhecer redes de apoio micro e macrossociais que possam auxiliá-los em suas demandas do cotidiano.

A não ocorrência de quedas nos últimos 12 meses e a ausência do indicativo de sintomas depressivos associaram-se ao melhor nível de apoio social, refletindo na perspectiva de uma relação positiva entre melhores condiçóes de saúde e a presença de algum tipo suporte nessa fase da vida.

\section{REFERÊNCIAS}

1. Miranda GMD, Mendes ACG, Silva ALA. O envelhecimento populacional brasileiro: desafios e consequências sociais atuais e futuras. Rev bras geriatra gerontol. [Internet] 2016 [citado 2019 mar 08]; 19(3): 507-19. Disponível em: http://dx.doi. org/10.1590/1809-98232016019.150140

2. Departamento de Informática do Sistema Único de Saúde-DATASUS. Projeçáo da população das unidades federaçáo por sexo e grupos de idade: 2000-2030. [Internet] 2019 [citado 2019 ago 13]. Disponível em: http://tabnet.datasus.gov.br/cgi/ deftohtm.exe?ibge/cnv/projpopuf.def.

3. Macedo JP, Dimenstein M, Sousa HR, Costa APA, Silva BIBM. A produção científica brasileira sobre apoio social: tendências e invisibilidades. Gerais: Rev Interinst Psicol. [Internet] 2018 [citado 2019 mar 10]; 11(2):258-278. Disponível em: http:// dx.doi.org/10.36298/gerais2019110206.

4. World Health Organization. Active Ageing: A Policy Framework. Geneva: WHO [Internet]. 2002 [citado em 2019 mar 08]. Disponível em: https://www.who.int/ageing/publications/active_ ageing/en/

5. Guedes MBOG, Lima KC, Caldas CP, Veras RP. Apoio social e o cuidado integral à saúde do idoso. Physis [Internet] 2017 [citado 2019 mar 08]; 27(4): 1185-1204. Disponível em: http://dx.doi. org/10.1590/s0103-73312017000400017.

6. Santini ZI, Fiori KL, Feeney J, Tyrovolas S, Haro JM, Koyanaji A. Social relationships, loneliness, and mental health among older men and women in Ireland: A prospective community-based study. J Affect Disord. [Internet] 2016 [citado 2019 mar 08]; 204: 59-69. Disponível em: http://dx.doi. org/10.1016/j.jad.2016.06.032.
Conhecer a relação entre apoio social e as condiçôes de saúde em idosos da comunidade é essencial para o planejamento e a implementação de um plano de cuidados adequado que garanta a utilização do apoio social como uma estratégia que beneficia a saúde dessa população. Assim, espera-se que a divulgação dos dados obtidos possa promover a discussão e favorecer o desenvolvimento de uma linha de cuidado em enfermagem ao idoso capaz de promover açóes destinadas a melhorar as condiçôes de saúde dessa população.

7. Susanto T, Bachtiar S, Turwantoko T. Performance of Public Health Nurses and Coverage of the Nursing Care Program by Community Health Centers in Jember, Indonesia. Int J Community Based Nurs Midwifery. [Internet] 2019 [citado 2019 fev 10]; 7(2): 161-168. Disponível em: http://dx.doi.org/10.30476/IJCBNM.2019.44887

8. Bertolucci PHF, Brucki SMD, Campacci SR, Juliano Y. O Mini-Exame do Estado Mental em uma população geral: impacto da escolaridade. Arq Neuro-Psiquiatr [Internet] 1994 [citado $2019 \mathrm{fev}$ 10]; 52(1): 01-07. Disponível em: https://www. scielo.br/pdf/anp/v52n1/01.pdf

9. Almeida OP, Almeida SA. Confiabilidade da versão brasileira da Escala de Depressão em Geriatria (GDS) versão reduzida. Arq Neuro-Psiquiatr. [Internet] 1999 [citado 2019 fev 10]; 57(2-B): 42126. Disponível em: http://dx.doi.org/10.1590/ S0004-282X1999000300013.

10. Nakano MM. Versáo brasileira Short Physical Performance Battery SPPB: adaptação cultural e estudo da confiabilidade [dissertação]. [Internet] Campinas: Faculdade de Educação, Universidade Estadual de Campinas; 2007 [citado 2019 fev 10]. Disponível em: http://repositorio.unicamp.br/ handle/REPOSIP/252485

11. Ramos LR, Perracini M, Rosa TE, Kalache A. Significance and management of disability among urban elderly residents in Brazil. J Cross-Cultural Gerontology. [Internet] 1993 [citado 2019 fev 10]; 8(4): 3313-23. Disponível em: https://doi. org/10.1007/BF00972560

12. Fried LP, Tangen CM, Walston J, Newman AB, Hirsch C, Gottdiener J et al. Frailty in older adults evidence for a phenotype. The Journals of Gerontology Series A: Biological Sciences and Medical Sciences [Internet] 2001 [citado 2019 June 07]; 56(3): 146-157. Disponível em: https:// 
doi.org/10.1093/gerona/56.3.M146.

13. Batistoni SST, Neri AL, Cupertino APFB. Validade da escala de depressão do Center for Epidemiological Studies entre idosos brasileiros. Rev Saúde Publica, São Paulo. 2007 [citado 2019 June 07]; 41(4): 598-605. Disponível em: https:// doi.org/10.1590/S0034-89102007000400014

14. Benedetti TRB, Mazo GZ, Barros MVG. Aplicação do questionário internacional de atividades físicas (IPAQ) para avaliação do nível de atividades físicas de mulheres idosas: validade concorrente e reprodutibilidade teste-reteste. $\mathrm{R}$ Bras $\mathrm{Ci}$ e Mov [Internet] 2004 [citado 2019 June 07]; 12(1): 2533. Disponível em: http://dx.doi.org/10.18511/ rbcm.v12i1.538.

15. Lebrão ML, Duarte YAO. SABE - Saúde, Bemestar e Envelhecimento - O Projeto Sabe no município de São Paulo: uma abordagem inicial -Brasília: Organização Pan-Americana da Saúde, p. 255, 2003.

16. Griep RH, Chor D, Faerstein E, Werneck GL, Lopes CS. Validade de constructo de escala de apoio social do Medical Outcomes Study adaptada para o português no Estudo Pró-Saúde. Cad Saúde Pública. 2005 [citado 2019 June 07]; 21(3): 703714. Disponível em: http://dx.doi.org/10.1590/ S0102-311X2005000300004

17. Coelho FF, Michel RB. Associação entre cognição, suporte social e qualidade de vida de idosos atendidos em uma unidade de saúde de Curitiba/ PR. Ciênc cogn. [Internet] 2018 [citado 2019 jun 07]; 23(1): 54-62. Disponível em: http://www. cienciasecognicao.org/revista/index.php/cec/article/ view/1349/pdf_100

18. Torres JL, Castro-Costa E, Mambrini JVM, Peixoto SWV, Diniz BSO, Oliveira C. et al. Sintomas depressivos, apoio emocional e início do comprometimento das atividades da vida diária: seguimento de 15 anos do Estudo de Coorte de Idosos de Bambuí, Minas Gerais, Brasil. Cad Saúde Pública. [Internet] 2018 [citado 2019 mar 08]; 34(7): e00141917. Disponível em: https://doi. org/10.1590/0102-311x00141917.

19. Departamento de Informática do Sistema Único de Saúde - DATASUS. Internaçóes por Unidade de Federação segundo faixa etária. Período de janeiro a agosto de 2018 [Internet] 2018 [citado 2018 nov 05]. Disponível em: http://tabnet.datasus.gov.br/ cgi/tabcgi.exe?sih/cnv/niuf.def

20. Teixeira JJM, Bastos GCFC, Souza ACL. Perfil de internação de idosos. Rev Soc Bras Clin Med. [Internet] 2017 [citado 2019 mar 08]; 15(1): 15-20. Disponível em: http://docs.bvsalud.org/ biblioref/2017/04/833048/15-20.pdf

21. Pimentel WRT, Pagotto V, Stopa SR, Hoffmann
MCCL, Malta DC, Menezes RL. Quedas com necessidade de procura de serviços de saúde entre idosos: uma análise da Pesquisa Nacional de Saúde, 2013. Cad Saúde Pública. [Internet] 2018 [citado 2019 mar 08]; 34(8): e00211417. Disponível em: https://doi.org/10.1590/0102-311x00211417.

22. Rosa TSM, Santos Filha VAV, Moraes AB. Prevalência e fatores associados ao prejuízo cognitivo em idosos de instituiçôes filantrópicas: um estudo descritivo. Ciênc saúde coletiva. [Internet] 2018 [citado 2019 mar 08]; 23(11): 3757-3765. Disponível em: https://doi.org/10.1590/1413812320182311.25212016

23. Duarte YAO, Nunes DP, Andrade FB, Corona LP, Brito TRP, Santos JLF et al. Fragilidade em idosos no município de São Paulo: prevalência e fatores associados. Rev Bras Epidemiol. [Internet] 2018 [citado 2019 mar 08]; 21(Suppl 2): E180021. Disponível em: https://doi.org/10.1590/1980549720180021.supl.2

24. Oliveira DC, Neri AL, D'Elboux MJ. Ausência de expectativa de suporte para o cuidado aos idosos da comunidade. Rev Bras Enferm [Internet]. 2016 [citado 2019 mar 08]; 69(3): 566-73. Disponível em: https://doi.org/10.1590/00347167.2016690321.

25. Moreno CRC, Santos JLF, Lebrão ML, Ulhôa MA, Duarte YAO. Problemas de sono em idosos estão associados a sexo feminino, dor e incontinência urinária. Rev Bras Epidemiol. [Internet] 2018 [citado 2019 mar 08]; 21(Supl 2): E180018. Disponível em: https://doi.org/10.1590/1980549720180018.supl.2.

26. Aragão EIS, Campos MR, Portugal FB, Gonçalves DA, Mari JJ. Fortes SLCL. Padróes de Apoio Social na Atenção Primária à Saúde: diferenças entre ter doenças físicas ou transtornos mentais. Ciênc saúde coletiva. [Internet] 2018 [citado 2019 mar 08]; 23(7): 2339-2350. Disponível em: https://doi. org/10.1590/1413-81232018237.21012016.

27. Brito TRP, Pavarini SCI. Relaçấo entre apoio social e capacidade funcional de idosos com alteraçôes cognitivas. Rev Latino-Am Enfermagem [Internet]. 2012 [citado 2019 mar 08]; 20(4): 1-9. Disponível em: http://dx.doi.org/10.1590/S010411692012000400007.

28. Eslami B, Di Rosa M, Barros H, Stankunas M, Torres-Gonzalez F, Ioannidi-Kapolou E et al. Lifetime abuse and perceived social support among the elderly: a study from seven European countries. Eur J Public Health. [Internet] 2018 [citado 2019 mar 08]; 27(4): 686-692. Disponível em: https:// doi.org/10.1093/eurpub/ckx047

29. López-Cerdá E, Carmona-Torres JM, RodríguezBorrego MA. Social support for elderly people over 
65 years in Spain. Int Nursing Review. [Internet] 2019 [citado 2019 mar 08]; 66(1): 104-111. Disponível em: https://doi.org/10.1111/inr.12468

30. Sànchez Rodrigues MM, De Jong Gierveld J, Buz J. Loneliness and the exchange of social support among older adults in Spain and the Netherlands. Ageing Soc. [Internet] 2014 [citado 2019 mar 08]; 34: 330-54. Disponível em: https://doi. org/10.1017/S0144686X12000839

31. Brito AMM, Camargo BV, Giacomozzi AI, Berri B. Representaçóes sociais do cuidado ao idoso e mapas de rede social. Liber [Internet] 2017 [citado 2019 mar 08]; 23(1): 9-22. Disponível em: http:// dx.doi.org/10.24265/liberabit.2017.v23n1.01.

32. Zanini DS, Peixoto EM, Nakano EC. Escala de Apoio Social (MOS-SSS): proposta de normatizaçáo com referência nos itens. Trends Psychol [Internet] 2018 [citado 2019 mar 08]; 26(1): 387-99. Disponível em: http://dx.doi. org/10.9788/tp2018.1-15pt.

33. França-Santos D, Oliveira AJ, Salles-Costa R, Lopes CS, Sichieri R. Diferenças de gênero e idade no apoio social e índice de massa corporal em adultos na Região Metropolitana do Rio de Janeiro,
Brasil. Cad Saúde Pública. [Internet] 2017 [citado 2019 mar 08]; 33(5): e00152815. Disponível em: https://doi.org/10.1590/0102-311x00152815.

34. Durbin L, Kharrazi RJ, Graber R, Mielenz TJ. Social support and older adult falls. Inj Epidemiol. [Internet] 2016 [citado 2019 mar 08]; 3(1): 1-4. Disponível em: https://doi.org/10.1186/s40621016-0070-y

35. Possatto JM, Rabelo DF. Condiçôes de saúde psicológica, capacidade funcional e suporte social de idosos. Rev Kairós. [Internet] 2018 [citado 2019 mar 08]; 20(2): 45-58. Disponível em: https://doi. org/10.23925/2176-901X.2017v20i2p45-58

36. Gariepy G, Honkaniemi H, Quesnel-Vallee A. Social support and protection from depression: systematic review of current findings in Western countries. BJPsych. [Internet] 2016 [citado 2019 mar 08]; 209(1): 284-293. Disponível em: https:// doi.org/10.1192/bjp.bp.115.169094

37. Carmona CF, Couto VVD, Scorsolini-Comin F. A experiência de solidáo e a rede de apoio social de idosas. Psicol estud. [Internet] 2014 [citado 2019 mar 08]; 19(4): 681-691. Disponível em: http:// dx.doi.org/10.1590/1413-73722395510. 